\title{
A flora no Biota-MS: montando o quebra-cabeça da biodiversidade de Mato Grosso do Sul
}

\author{
Maria Ana Farinaccio', Fabio de Oliveira Roque', Gustavo Graciolli', \\ Paulo Robson de Souza' \& João Onofre Pereira Pinto ${ }^{2}$
}

\begin{abstract}
Universidade Federal de Mato Grosso do Sul, Centro de Ciências Biológicas e da Saúde, Cidade Universitária, Caixa Postal 549 CEP 79070-900, Campo Grande, MS, Brasil. mafarinaccio@hotmail.com

${ }^{2}$ Universidade Federal de Mato Grosso do Sul, Laboratório de Inteligência Artificial, Cidade Universitária, Caixa Postal 549. CEP 79070-900, Campo Grande, MS, Brasil
\end{abstract}

Recebido em 27.XI.2014

Aceito em 14.VII.2015

DOI 10.21826/2446-8231201873s11

\begin{abstract}
RESUMO - O número especial Biota-MS está constituído de 40 artigos sobre a biodiversidade da flora de Mato Grosso do Sul (elaborados por 112 pesquisadores de 39 instituições brasileiras e internacionais), em que são listadas 3.911 espécies, sendo discriminadas as espécies ameaçadas, endêmicas ou só citadas para o estado. Apesar dos entraves à preparação desta edição, tais como a falta de especialistas em diversos táxons e a dificuldade em quantificar a diversidade de espécies de Mato Grosso do Sul a partir do material coletado antes de 1977, quando Mato Grosso e Mato Grosso do Sul eram estado uno, os resultados obtidos constituem-se em um dos mais atualizados e sistematizados trabalhos de organização de dados biológicos no Brasil.
\end{abstract}

Palavras-chave: Cerrado, Chaco, Floresta Atlântica, Pantanal

ABSTRACT - The flora in Biota-MS: Assembling the puzzle of biodiversity of Mato Grosso do Sul state. The Biota-MS, a special issue of Iheringia, Série Botânica, consists of 40 articles on the biodiversity of Mato Grosso do Sul state, Brazil. It was written by 112 researchers from 39 Brazilian and international institutions. A total of 3,911 species were recorded, including endangered species and endemics to Mato Grosso do Sul or those only cited for MS. Despite obstacles in the preparation of this edition, such as the lack of specialists in different taxa and the difficulty in quantifying species diversity of Mato Grosso do Sul from material collected before 1977, when Mato Grosso and Mato Grosso do Sul states had not been split, the results obtained are one of the most updated and systematized work of organizing biological data in Brazil.

Keywords: Cerrado, Chaco, Tropical Atlantic rain forest, Pantanal

\section{Por que montar o quebra-cabeça?}

A Convenção sobre Diversidade Biológica (Convention on Biological Diversity - CBD 1992) define biodiversidade ou diversidade biológica como "a variabilidade entre organismos vivos de qualquer origem incluindo, entre outros, ecossistemas terrestres, marinhos e outros ecossistemas aquáticos, e os complexos ecológicos de que fazem parte". Em síntese, ela abrange três dimensões: (i) a diversidade de espécies, também denominada diversidade entre espécies, expressa o número e a abundância relativa de espécies em uma determinada comunidade de organismos; (ii) a diversidade intraespecífica, que abrange variações morfológicas, fisiológicas e de comportamento entre indivíduos de uma população ou entre populações espacialmente distintas da mesma espécie - esta, por incluir diferenças de algum modo derivadas da ação gênica, também é denominada diversidade genética; e (iii) a diversidade de ecossistemas, mais relacionada aos processos funcionais e que, em termos práticos, segundo Lewinsohn (2006) tem sido tratada como correlacionada com a diversidade de fisionomias de vegetação, de paisagens ou de biomas. Entretanto, o autor destaca que, no campo social e político, biodiversidade assumiu outros significados que extrapolam as questões essencialmente científicas.
A diversidade biológica, além do seu valor intrínseco às espécies, aqui consideradas não apenas como categoria taxonômica, mas como unidades portadoras de patrimônio genético alicerçado em uma história evolutiva, possui inestimável valor para a sobrevivência humana. Além dos benefícios ambientais que proporciona, como por exemplo, a purificação da água, a ciclagem de nutrientes, a manutenção do equilíbrio dinâmico dos ecossistemas e das condições climáticas da Terra, constitui uma base de recursos de aplicação alimentar, medicinal e industrial, entre outras (Millennium Ecosystem Assessment 2005).

É consenso que o Brasil tem posição chave na manutenção da biodiversidade do planeta, devido à sua riqueza natural - uma das mais megadiversas no mundo, o que corresponde entre 15 e $20 \%$ das espécies conhecidas (Lewinsohn \& Prado 2005).

A complexidade da tarefa de descobrir, descrever, caracterizar e fazer bom uso dos produtos derivados da diversidade biológica brasileira, assim como de entender padrões de mudanças da estrutura e função da biodiversidade e seus impactos na sociedade humana, exige um esforço científico cooperativo e articulado. Assim, o estabelecimento e a manutenção de uma agenda de pesquisa de longo prazo em biodiversidade constituem um desafio estratégico para o Brasil. 
Nosso conhecimento sobre biodiversidade no mundo ainda é bastante rudimentar, pois a maioria das espécies não está formalmente descrita ou suas distribuições geográficas são mal definidas e entendidas (Bini et al. 2006). Quaisquer estratégias de conservação, monitoramento e uso sustentável da biodiversidade dependem destas informações e, como vários fatores provenientes das ações humanas atuam sinergeticamente na degradação de ambientes aquáticos e terrestres, a perda de espécies está em ritmo mais acelerado do que o ritmo de ampliação do conhecimento sobre biodiversidade. Fortes investimentos são requeridos em níveis global, regionais e locais para mitigar esta situação (Convention on Biological Diversity CBD 1992, Millenium Ecossistem Assessment 2005). Um importante exemplo de iniciativas destes investimentos foi a própria $\mathrm{CBD}$, que tem por objetivos a conservação e a utilização sustentável da biodiversidade e a repartição justa e equitativa dos benefícios decorrentes de sua utilização, bem como dos conhecimentos tradicionais associados. Recentemente, na cidade de Nagoya, Japão, durante a Conferência das Partes 10 foi acordado entre os países que integram a CDB atender as 20 metas do Plano Estratégico 2011-2020, conhecidas como Metas de Aichi, todas voltadas à redução da perda da biodiversidade em âmbito mundial.

Embora ainda de forma deficiente, o Brasil direciona esforços para atingir suas metas de redução da perda da biodiversidade e segue a forte tendência mundial de integrar informações biológicas em sistemas colaborativos e de fácil acesso, que possam subsidiar tomadas de decisão. Um exemplo claro foi a criação, pelo governo brasileiro, do Programa Nacional da Diversidade Biológica PRONABIO, instituído pelo Decreto n ${ }^{\circ} 1.354$ de 29 de dezembro de 1994, para coordenar a implementação dos compromissos da CDB. Conforme sintetizado por Lewinsohn \& Prado (2005), no Brasil existe forte discrepância no conhecimento da biodiversidade por regiões, sendo as regiões Centro-Oeste e Nordeste particularmente carentes de investimentos e dados sobre a fauna e a flora. As metas da CBD ou qualquer outra meta de conhecimento e redução de perda de biodiversidade, só serão atingidas se melhorarmos a representatividade e a qualidade dos dados sobre biodiversidade em diversos níveis, se lacunas de conhecimento existentes forem preenchidas focando em regiões pouco estudadas e se houver forte investimento em infraestrutura e formação de pessoal em instituições locais.

A conservação e o uso sustentável da biodiversidade são questões complexas e demandam esforços integrados em longo prazo, principalmente em países megadiversos como o Brasil. Logicamente, a melhor estratégia para esta superação é o investimento em estudos sobre biodiversidade em diferentes dimensões. Entretanto, mesmo com aumento de iniciativas em larga escala, parcialmente impulsionadas por novas tecnologias (Snaddon et al. 2012), ainda serão necessárias muitas décadas para a descrição de espécies em grupos hiperdiversos. Mora et al. (2011) estimam que existam na biosfera 8,7 milhões de espécies, desconsiderados os vírus e bactérias, sendo que $86 \%$ dessas permanecem sem nomes, fato que permite uma noção do imenso desconhecimento sobre a história natural e biologia das espécies, assim como sobre os possíveis usos da biodiversidade.

Portanto, é essencial a elaboração de amplas estratégias de pesquisa e formação de pessoal que envolvam a obtenção de informações sobre a biodiversidade (e.g. taxonômicas e distribucionais). A obtenção desses dados deve permitir a avaliação dos correntes impactos antrópicos na biodiversidade, das formas de monitoramento de estratégias de conservação em níveis locais, regionais, nacionais e internacionais e, principalmente, do uso sustentável desta biodiversidade. Entretanto, o primeiro passo para se montar o quebra-cabeça da biodiversidade, aqui considerada no conceito amplo (Lewinsohn 2006), é conhecer a diversidade de espécies e sua distribuição, base primordial para essas ações.

\section{O tabuleiro: biodiversidade como um tema estratégico para o estado de Mato Grosso do Sul}

O reconhecimento dos múltiplos valores da biodiversidade - particularmente os econômicos - começou com a observação de que quanto mais diversidade de vida um país possui, uma gama maior de produtos podem ser desenvolvidos e industrializados. Tomando-se como exemplo o Brasil, estima-se que aproximadamente $45 \%$ do produto interno bruto seja derivado da utilização de recursos da biodiversidade, incluindo produtos da agroindústria, setor florestal e pesca, aqui desconsiderados os serviços ambientais.

Com 357.145,532 km² e 2.449.024 habitantes (IBGE 2013), Mato Grosso do Sul encontra-se numa região estratégica em termos de biodiversidade (Fig. 1), onde ocorre o contato entre vários macroecossistemas: Cerrado, Chaco, Floresta Chiquitana, Floresta Atlântica, Floresta Amazônica. Cerradão é a fitofisionomia mais comum no estado, enquanto que cerrado stricto sensu e cerrado arbustivo frequentemente ocorrem em terrenos rochosos ou mal drenados. Na parte sul ocorre a Floresta Atlântica de interior ou semidecidual, ou da bacia do Paraná-Paraguai. Florestas secas são encontradas em afloramentos calcários ou basálticos. Savanas inundáveis monodominantes são encontradas no Pantanal, um mosaico de várias influências fitogeográficas (Pott et al. 2012).

Esta localização peculiar e os tipos vegetacionais nele encontrados resultam em uma diversidade biológica relativamente alta, e algumas regiões podem ser consideradas únicas no Brasil, como aquela sob influência direta do Chaco e da Floresta Chiquitana da Bolívia. Várias espécies ocorrem no país, exclusiva ou quase exclusivamente, em território sul-mato-grossense, em função desta peculiaridade, tais como Zamia boliviana (Brongn.) A. DC., Zamiaceae quase ameaçada (IUCN 2013); Mandevilla angustifolia (Malme) Woodson, Apocynaceae (Fig. 2D, primeira citação para o Brasil); Dimerostemma annuиm (Hassl.) H. Rob., 


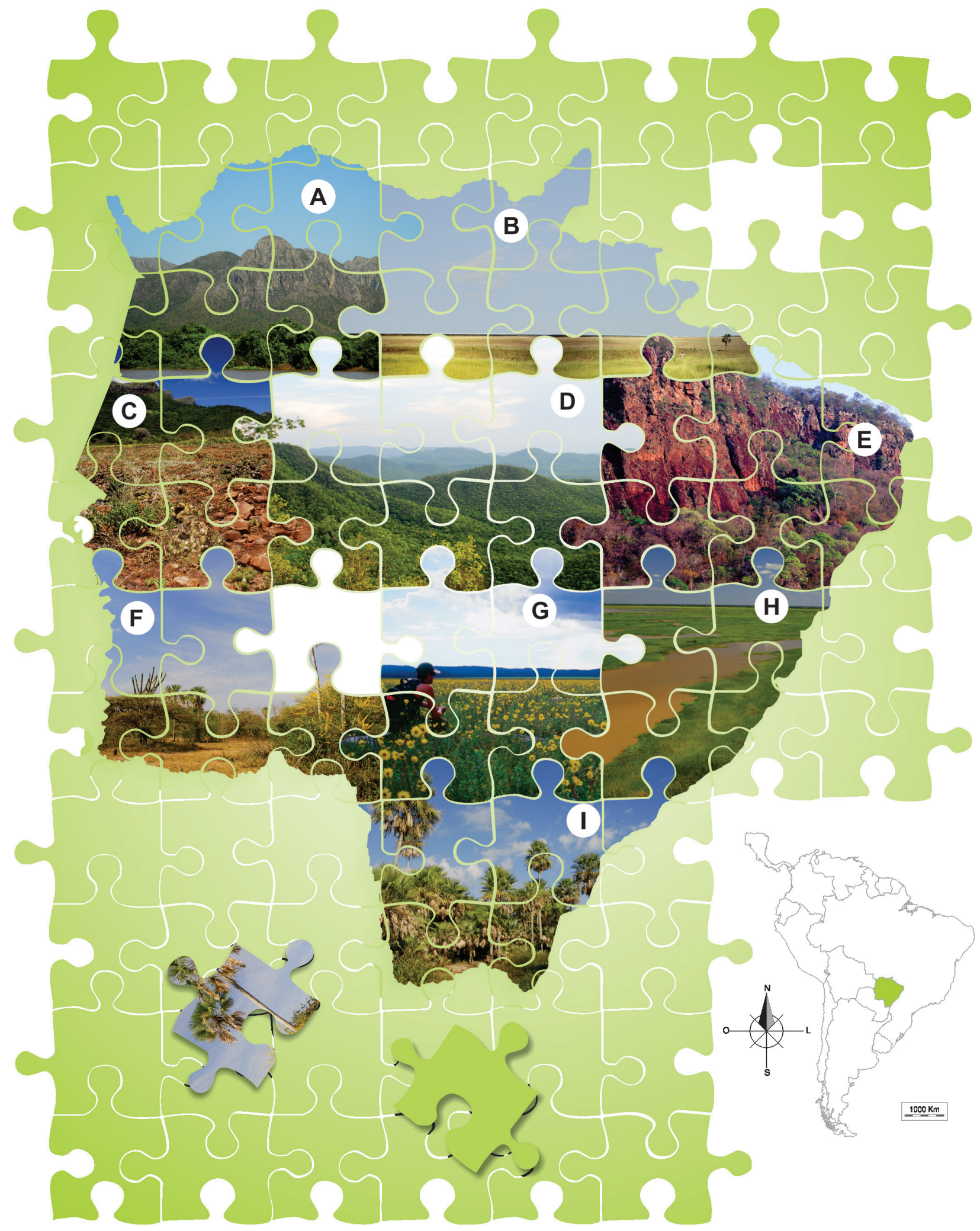

Figs. 1 A-I. Paisagens e ambientes de Mato Grosso do Sul. A. Serra do Amolar; B. Coval, nascente do Sucuriú, com Mauritia flexuosa à direita (V. J. Pott); C. Bancada laterítica com Discocactus ferricola em primeiro plano e morraria do Urucum ao fundo; D. Planalto da Bodoquena; E. Borda oeste do planalto de Maracaju, em Piraputanga, com floresta estacional decidual; F. Fitofisionomia chaquenha do tipo Chaco arborizado, município de Porto Murtinho; G. Lagoa das Pedras, mun. de Bonito, com predomínio de Dimerostemma annuum, registro de março de 2000; H. Planície alagada (Pantanal) com arroz nativo, prox. à foz do rio São Lourenço; I. Carandazal (Copernicia alba), mun. Porto Murtinho. Barra $=1000$ Km. Nem todas as imagens coincidem com a localização no mapa. 




Figs. 2 A-F. Espécies endêmicas de Mato Grosso do Sul e entorno ou, no Brasil, só citadas para o Mato Grosso do Sul. A. Stetsonia coryne; B. Opuntia elata; C. Prosopis rubriflora (A. B. Sartori); D. Mandevilla angustifolia; E. Tillandsia bonita (L. M. Versieux); F. Aspilia grazielae.

Asteraceae [Figura 1G, descrita em 1915 para o Paraguai e reencontrada 85 anos depois, no município de Bonito ver Moraes \& Semir (2009)]; Prosopis rubriflora Hassler, Leguminosae (exclusiva do Chaco brasileiro, Fig. 2C); Stetsonia coryne (Salm-Dyck) Britton \& Rose (Fig. 2A) e Opuntia elata Link \& Otto ex Salm-Dyck, Cactaceae (ver Zappi et al., nesta edição).

O estado também abriga espécies endêmicas, como Gomphrena centrota E. Holzh., Amaranthaceae; Aspilia grazielae J.U. Santos, Asteraceae (Fig. 2F) - ambas 
em risco de extinção; Discocactus ferricola Buining \& Brederoo (Figura 1C), Cactaceae de distribuição restrita (Martinelli \& Moraes 2013). Dentre as espécies novas ou recentemente descritas está Tillandsia bonita Versieux \& Martinelli (Fig. 2E), Bromeliaceae (Versieux et al. 2013).

A biodiversidade tem sido a base da economia em várias regiões de Mato Grosso do Sul, como no Pantanal, onde a pecuária se apoia basicamente na diversidade da flora nativa, sendo os campos naturais o principal recurso forrageiro da região. O turismo no Pantanal e na região da Serra da Bodoquena utiliza a biodiversidade como o mais forte atrativo, incluindo pesca desportiva e a contemplação da fauna e da paisagem.

A biodiversidade também está fortemente ligada ao componente sociocultural. A região sul-mato-grossense é marcada pela presença de aldeias indígenas e quilombolas que historicamente construíram formas diversas de interação com a natureza e que vivenciam crescente demanda por iniciativas coletivamente construídas, que possam subsidiar programas de gestão territorial e manejo de recursos sustentáveis.

A região também é marcada pela presença de movimentos sociais relacionados à reforma agrária. A presença de comunidades quilombolas também é reconhecida em várias regiões do estado. Estes movimentos elaboram, junto às famílias, desejos de recriação de formas tradicionais de produção e de identidade com a terra, o que as levam à valorização do ambiente que ocupam. Tal valorização tem sido profícua numa relação de forma integrada, envolvendo a valorização dos saberes tradicionais (cultura), bem como a valorização da vida (pessoas, ambiente e biodiversidade). Tais organizações sociais demandam iniciativas e estratégias de usos sustentáveis de seus recursos.

Enfim, o tema "biodiversidade" está diretamente relacionado às dimensões socioambientais, políticas, econômicas e educacionais e, regionalmente, demanda iniciativas consistentes e de longo prazo como a proposta do Programa Biota-MS.

\section{O que é o Biota-MS?}

Em 2007, o Governo do estado de Mato Grosso do Sul, por meio da Superintendência de Ciência e Tecnologia (Sucitec-MS), vinculada à Secretaria de estado de Meio Ambiente, do Planejamento, da Ciência e Tecnologia, submeteu o projeto "BIOECONOMIA - novo paradigma de desenvolvimento para Mato Grosso do Sul" à Chamada Pública da Financiadora de Estudos e Projetos - FINEP (Edital Estruturante em C\&T). Em dezembro de 2008, o Governo do estado de Mato Grosso do Sul e a FINEP firmaram convênio para implementar o projeto. A partir dessa data, a Sucitec-MS optou pela ampliação dos dois subprojetos que estão sob sua responsabilidade: o Bioenergia e o Biota-MS. Para tanto, iniciou um processo de planejamento participativo e convidou as instituições de ensino e pesquisa e a comunidade para debater o subprojeto, inspirados nos moldes do Programa BIOTA/FAPESP.
Especificamente para o Biota-MS, em 2009 foram realizados dois seminários para discutir as bases para sua implantação, em que a Sucitec-MS apresentou o subprojeto e propôs sua ampliação para um programa, considerando a importância do tema para o estado. Nesse evento, foram consolidadas as primeiras ações para o desenvolvimento do Programa Biota-MS, sendo a primeira delas a constituição de um comitê gestor temporário. Este comitê foi o responsável pela ampliação do subprojeto para um "Programa de Ciência, Tecnologia \& Inovação em Biodiversidade de Mato Grosso do Sul (Biota-MS)".

O Programa Biota-MS tem a missão de construir uma base integrada de conhecimentos científicos, tecnológicos e de inovação no Mato Grosso do Sul para dar suporte a tomadas de decisão em gestão da biodiversidade. Tem como visão, ser um programa e organização de referência na integração de iniciativas e projetos que promovam o entendimento sobre a biodiversidade, seu uso e conservação no estado. Seus valores são a interdisciplinaridade, a colaboração, a transparência, a eficácia, a excelência em gestão e a divulgação do conhecimento de biodiversidade.

\section{As regras e participantes do jogo}

Para atingir sua missão, o programa trabalha em rede, articulando pessoas e infraestrutura de forma colaborativa, sob a coordenação da Sucitec-MS. Na sua origem, o programa contou com o apoio institucional de universidades e institutos de pesquisa do estado. Ao longo de seu desenvolvimento, a rede visa ampliar a integração de diferentes setores sociais, nacional e internacionalmente.

Durante os seminários foi definido como ação prioritária levantar o conhecimento disponível sobre a biodiversidade no estado. Para cumprir tal objetivo foi constituído um grupo de pesquisadores para organizar as listas de espécies da flora e fauna conhecidas no estado. Com o objetivo de inventariar a biodiversidade do estado conhecida até o momento (informação publicada ou disponível em coleção científica) em um volume especial, financiado pelo Governo de estado de Mato Grosso do Sul, os pesquisadores decidiram montar uma rede de colaboradores que seria formada por especialistas residentes e não residentes no estado de Mato Grosso do Sul para contribuir com as listagens de espécies de sua competência. Estes especialistas tiveram a liberdade de convidar novos colaboradores para serem coautores de seus trabalhos e indicar outros para grupos taxonômicos onde não havia colaborador.

\section{Desafios na obtenção de algumas peças}

Dentre os desafios enfrentados pelos participantes deste jogo, principalmente para encaixar as peças soltas, apesar da equipe da flora ter as coleções botânicas totalmente informatizadas, ou em franco avanço no processo de informatização, os botânicos têm à disposição a integração de imagens de plantas e fungos coletados no Brasil, mantidos em herbários do país e do exterior, aos dados disponíveis na rede INCT-Herbário Virtual da Flora e dos Fungos via speciesLink. 
No entanto, nesse tabuleiro, os especialistas se deparam com um desafio maior, a divisão do estado em 1977, que antes fazia parte de Mato Grosso. Coleções referidas para o estado de Mato Grosso uno, portanto antes da divisão, nem sempre foram checadas e adequadas à nova divisão geopolítica do país, subestimando o número de espécies que ocorrem em Mato Grosso do Sul. Esse fato pode ser facilmente identificado, já que nos rótulos de coletas realizadas em Mato Grosso do Sul antes da divisão, consta "estado de Mato Grosso" e o desconhecimento dos municípios de cada um dos estados leva a incorreções. Um exemplo seria Matelea matogrossensis Fontella, que foi descrita com base em um material proveniente de Terenos, MS [Hatchbach 24617 (MBM)] e, posteriormente coletada em Rio Brilhante (MS) não foi citada para o Mato Grosso do Sul na Lista de Espécies da Flora do Brasil (Koch et al. 2012), porque esses municípios foram interpretados como pertencentes ao estado de Mato Grosso (ver Farinaccio \& Simões, nesta edição). Na montagem desse jogo, o checklist das espécies de Angiospermas de Dubs (1998) pode representar uma armadilha e suas peças continuarem soltas se a distribuição das espécies não foi devidamente atualizada. Em suma, para juntar as peças nesse tabuleiro, todos enfrentaram um mesmo desafio, sistematizar os dados dispersos, organizá-los e encaixá-los e, assim, saber o que já se conhece do estado de Mato Grosso do Sul.

Entretanto, o maior de todos os desafios, em um país megadiverso como o Brasil, é trabalhar com a falta de especialistas em todos os grupos, ou ainda ter especialistas com disponibilidade de aceitar tal tarefa. Este é um dos motivos pelos quais a presente publicação não trata da microbiota, bem como não abrange todas as famílias de plantas e animais existentes em Mato Grosso do Sul.

\section{Peças encaixadas e peças ainda soltas}

Nesta edição são apresentados 40 artigos sobre a biodiversidade vegetal de Mato Grosso do Sul. São 112 autores de 39 instituições brasileiras e internacionais (Bolívia, Equador, França, Reino Unido e Singapura), sendo 27, vinculados a instituições de ensino e/ou pesquisa e órgãos de MS. O número de colaboradores locais demonstra um amadurecimento dos grupos de pesquisa no estado e o contínuo fortalecimento e ampliação dos seus cursos de pós-graduação e centros de pesquisa.

Além das listas por grupos taxonômicos, são apresentadas listagens de espécies encontradas em ambientes que se destacam pela sua exclusividade e fragilidade, ou ainda associadas ao conhecimento tradicional (e.g. plantas alimentícias em Bortolloto et al., 2015).

Ao longo dos artigos são registradas 3.911 espécies da flora em Mato Grosso do Sul (Tabelas 1,2). Este número coloca o estado entre aqueles com organização mais atualizada e sistematizada de dados biológicos no Brasil como marco inicial do programa Biota-MS.
Tabela 1. Número de espécies por grupo taxonômico, da flora no estado de Mato Grosso do Sul, Brasil.

\begin{tabular}{lc}
\hline Grupo Taxonômico & Número de Espécies \\
\hline Charophyceae & 16 \\
Fitoplâncton & 940 \\
Pteridophyta & 225 \\
Angiospermas & 2.730 \\
\hline Total & 3.911 \\
\hline
\end{tabular}

Tabela 2. Número de espécies de Angiospermas por ordem ou família, no estado de Mato Grosso do Sul, Brasil.

\begin{tabular}{|c|c|}
\hline Ordem / Família & Número de Espécies \\
\hline Alismataceae & 23 \\
\hline Annonaceae & 19 \\
\hline Apiales & 21 \\
\hline Apocynaceae & 122 \\
\hline Araceae & 10 \\
\hline Asteraceae & 335 \\
\hline Begoniaceae & 5 \\
\hline Bignoniaceae & 57 \\
\hline Bromeliaceae & 41 \\
\hline Burseraceae & 9 \\
\hline Cabombaceae & 2 \\
\hline Cactaceae & 32 \\
\hline Capparaceae & 12 \\
\hline Cucurbitaceae & 27 \\
\hline Cyperaceae & 183 \\
\hline Erythroxylaceae & 12 \\
\hline Euphorbiaceae s.s. & 153 \\
\hline Moraceae & 12 \\
\hline Hydrocharitaceae & 7 \\
\hline Lauraceae & 26 \\
\hline Leguminosae & 423 \\
\hline Malpighiaceae & 114 \\
\hline Melastomataceae & 58 \\
\hline Meliaceae & 24 \\
\hline Myrtaceae & 81 \\
\hline Nymphaeaceae & 10 \\
\hline Orchidaceae & 150 \\
\hline Oxalidaceae & 17 \\
\hline Peraceae & 2 \\
\hline Phyllanthaceae & 16 \\
\hline Picraminales & 2 \\
\hline Plumbaginaceae & 1 \\
\hline Poaceae & 439 \\
\hline Podostemaceae & 3 \\
\hline Polygonaceae & 27 \\
\hline Potamogetonaceae & 3 \\
\hline Rubiaceae & 154 \\
\hline Rutaceae & 24 \\
\hline Sapindales & 66 \\
\hline Simaroubaceae & 8 \\
\hline Total & 2.730 \\
\hline
\end{tabular}

\section{Desdobramentos da figura que começa ser formada pelo quebra-cabeça}

O desconhecimento da identidade e distribuição dos organismos é um impedimento não apenas para entender padrões de biodiversidade, mas também para 
geri-la. Portanto, as listagens de espécies ocorrentes em determinadas áreas geográficas ou políticas são prérequisitos para diagnosticar o estado do conhecimento, indicar lacunas e desenhar novas estratégias de pesquisa e conservação.

Os resultados apresentados nesta edição, somados ao volume especial da Revista Iheringia, Série Zoologia, são requisitos para o melhor uso de informações sobre biodiversidade em políticas de públicas e instrumentos de gestão, por exemplo, em estudos de impacto ambiental, zoneamento ecológico econômico e biomonitoramento. As listagens também serão essenciais para o processo de avaliação do status de conservação das espécies e conhecimento de espécies ameaçadas, para embasar diversas linhas de pesquisa em ecologia e, principalmente, para o desenvolvimento de planos de ação para conservação da biodiversidade em Mato Grosso do Sul. Destacamos ainda, o potencial de utilização dos dados em programas de educação alicerçados em realidades locais e regionais.

Em síntese, consideramos esta iniciativa - do Programa Biota-MS - de compilar os dados existentes sobre a biodiversidade de Mato Grosso do Sul o nosso ponto de partida para monitorarmos os avanços do conhecimento sobre e para a biodiversidade nos próximos anos no estado, tanto no contexto do entendimento científico, como no seu uso em gestão e conservação da biodiversidade.

\section{AGRADECIMENTOS}

Agradecemos à FINEP pelo apoio financeiro ao desenvolvimento do programa Biota-MS. Ao Programa Zoneamento Ecológico Econômico de Mato Grosso do Sul - ZEE/MS, à Fundação de Apoio ao Desenvolvimento do Ensino, Ciência e Tecnologia do Estado de Mato Grosso do Sul - Fundect/MS, à Sucitec-MS e à Fundação Neotrópica do Brasil pelo convite de participação neste volume especial e o suporte financeiro para sua publicação. À Dra. Lezilda Carvalho Torgan, editora chefe da Revista Iheringia, Série Botânica e a todos os pesquisadores, técnicos e demais profissionais envolvidos neste projeto. Ao Dr. Arnildo Pott, pela revisão dos textos em inglês.

\section{REFERÊNCIAS}

Barcode of Life Data Systems. 2013. Barcode of Life. Disponível em : http:// www.barcodinglife.org. Acessado em 13.04.2013.

Bini, L.M., Diniz-Filho, J.A.F., Rangel, T.F.L.V.B., Bastos, R.P. \& Pinto, M.P. 2006. Challenging Wallacean and Linnean shortfalls: knowledge gradients and conservation planning in a biodiversity hotspot. Diversity Distrib. 12:475-482.

Bortolloto, I.M., Damasceno-Júnior, G.A. \& Pott, A. 2015 Lista preliminar das plantas alimentícias nativas de Mato Grosso do Sul, Brasil. Iheringia. Série Botânica 73(supl.):101-106.

Convention on Biological Diversity - CBD. 1992. Conference on Environment and Development, 5 June 1992, Rio de Janeiro. Disponível em: http:/www.biodiv.org. Acessado em 10.04.2011.

Dubs, B. 1998. Prodromus florae Matogrossensis. Part 1. Checklist of angiosperms. The botany of Mato Grosso, ser. B, no. 3. Kusnacht, Suíça.

Farinaccio, M.A. \& Simões, A.O. 2015. Checklist das Apocynaceae do Estado do Mato Grosso do Sul, Brasil. Iheringia. Série Botânica 73(supl.):131-146.

Global Biodiversity Information Facility - GBIF. 2013. Free and Open Access to Biodiversity Data http://www.gbif.org. Acessado em 10.03.2013

Instituto Brasileiro de Geografia e Estatística - IBGE. 2013. Estados@: Mato Grosso do Sul. Disponível em http://www.ibge.gov.br/estadosat/ perfil.php?sigla=ms. Acessado em 15.07.2013.

International Union For Conservation Of Nature And Natural Resources (IUCN). 2013. IUCN Red List of Threatened Species. Version 2013.1. Disponível em: http://www.iucnredlist.org/. Acessado em 21.10.2011.

Koch, I., Rapini, A., Kinoshita, L.S., Simões, A.O. \& Spina, A.P. 2012. Apocynaceae. In Lista de Espécies da Flora do Brasil. Jardim Botânico do Rio de Janeiro. p. 617-643.

Lewinsohn, T. (org.). 2006. Avaliação do estado do conhecimento da biodiversidade brasileira. Vol I. Brasília, Ministério do Meio Ambiente. 520p.

Lewinsohn, T.M. \& Prado, P.I. 2005. How many species are there in Brazil? Conservation Biology 19: 619-624.

Martinelli, G. \& Moraes, M.A. 2013. Livro vermelho da flora do Brasil. Jardim Botânico do Rio de Janeiro. http://cncflora.jbrj.gov.br. Acessado em 18.01.2013.

Millenium Ecosystem Assessment. 2005. Ecosystems and human wellbeing: synthesis. Island Press, Washington DC. Dispon http://www. millenniumassessment.org/documents/document.356.aspx.pdf.

Mora, C., Tittensor, D.P., Adl, S., Simpson, A.G.B. \& Worm, B. 2011. How many species are there on earth and in the ocean? PLoS Biol 9(8)e1001127:1-8.

Moraes, M.D \& Semir, J. 2009. A revision of Brazilian Dimerostemma (Asteraceae, Heliantheae, Ecliptinae), with a new species and taxonomic adjustments. Brittonia 61(4):341-365.

Pott, A., Pott, V.J. \& Moreira, S.N. 2012. Flora and vegetation of Mato Grosso do Sul, Brazil. Glalia 4(1):19-50.

Programa Biota/Fapesp. 2013. Instituto Virtual da Biodiversidade. Disponível em: http://www.biota.org.br. Acessado em 10.10.2012.

Snaddon J, Petrokofsky G, Jepson P \& Willis K.J. 2013 Biodiversity technologies: tools as change agents. Biol Lett 9: 20121029

SpeciesLink: "Sistema de Informação Distribuído para Coleções Biológicas: a Integração do Species Analyst e do SinBiota (FAPESP)". Banco de dados. Disponível em: http://www.splink.org.br/index. Acessado em 28.06.2013.

The Global Network for Taxonomy - BioNET. 2013. Disponível em: BioNet International Facility http://www.bionet-intl.org. Acessado em 15.05.2013.

Versieux, L.M., Vasconcellos, N. \& Martinelli. G. 2013. Tillandsia bonita (Bromeliaceae), a new species from Mato Grosso do Sul, Brazil, with notes on leaf anatomy. Systematic Botany 38(1):75-81.

Zappi, D.C, Taylor, N.G., Damasceno-Júnior, G.A., Pott, V.J. \& Machado, M.C. 2013. Checklist das Cactaceae do Estado do Mato Grosso do Sul, Brasil. Iheringia. Série Botânica 73(supl.):169-173. 